\title{
Neural stem/progenitor cells maintained in vitro under different culture conditions alter differentiation capacity of monocytes to generate dendritic cells
}

\author{
Lupatov $\mathrm{AYu}^{1}$, Poltavtseva $\mathrm{RA}^{2}$, Bystrykh $\mathrm{OA}^{2}$, Yarygin $\mathrm{KN}^{1}$, Sukhikh $\mathrm{GT}^{2}$.
}

\begin{abstract}
Cell therapy of the nervous system disorders using neural stem/progenitor cells (NSPCs) proved its efficacy in preclinical and pilot clinical studies. The mechanisms of the beneficial effects of NSPCs transplantation include replacement of damaged cells, paracrine activation of the regeneration, and immunomodulation. Detailed assessment of NSPCs-induced immunomodulation can contribute to better control of autoimmune reactions and inflammation in patients with neurodegenerative diseases. Interactions of NSPCs with dendritic cells (DCs), the key players in the induction of the immune system response to antigens are of particular interest. Here, we demonstrate that co-culturing of monocytes with NSPCs obtained and grown utilizing serum-containing medium instead of growth factor-containing serum-free medium, results in total suppression of monocyte differentiation into DCs. The effect is similar to the action of mesenchymal stem cells (MSCs). No significant effect on DCs maturation was observed. Cultures of NSPCs set up and maintained in serum-free medium have no influence on monocyte differentiation and DCs maturation. Therefore, the effects of NSPCs upon DC differentiation from monocytes strongly depend on culture conditions, whereas the molecular marker expression patterns are similar in both types of NSPCs cultures. In broader prospective, it means that cells with almost identical phenotypes can display opposite immunological properties depending upon culture conditions. It should be taken into account when developing NSPCs-based cell products for regenerative medicine.
\end{abstract}

Key Words: Neural stem cells, Neural progenitor cells, Immunomodulation, Dendritic cells, Cell therapy

\section{Introduction}

Allogeneic NSPCs have great potential as prospective therapeutic agents, due to low immunogenicity ${ }^{[1,2]}$ and the ability to stimulate restoration of the nervous tissue via paracrine mechanisms ${ }^{[3]}$. Moreover, it has been suggested that NSPCs, like MSCs, can suppress certain immunological reactions ${ }^{[4,5]}$. In vivo data and coculture experiments demonstrated NSPCs' capability to inhibit a number of immunological processes including proliferation and activation of T-cells in response to CNS-derived antigens or nonspecific polyclonal stimuli ${ }^{[6,7]}$, as well as pro-inflammatory signal molecule expression ${ }^{[8]}$. They are also able to reduce the number of memory cells and to increase the amount of IL-4 and IL-10 secreting CD4 positive T-cells ${ }^{[7]}$. NSPCs strongly suppress inflammation in the experimental autoimmune encephalomyelitis model ${ }^{[5,9]}$. Immunomodulation is probably the main mechanism of the healing effects of NSPCs transplantation in neuroinflammatory and autoimmune neurodegenerative diseases. In some cases, NSPCs may exert their action at the peripheral lymphoid organs level, without even entering the central nervous system ${ }^{[7,9]}$.

Dendritic cells (DCs) are a crucially important type of immune cells contributing to the development of the immune response to autologous and foreign antigens by efficient antigen capture, processing and presenting to lymphocytes. The advent of functionally active DCs includes two stages. At the first stage differentiation of CD34 positive bone marrow precursors or circulating monocytes delivers immature DCs exhibiting high phagocytic activity. Their interaction with lymphocytes not only fails to induce immune response, but can initiate the lymphocytes' anergy or tolerance to antigens ${ }^{[10]}$. In the presence of the factors of inflammation, immature DCs enter the second, maturation stage including the reduction of phagocytic activity ${ }^{[11,12]}$, enhancement of the expression of the major histocompatibility complex (MHC) and co-stimulation molecules, and the onset of the expression of chemokine receptors guiding DCs' migration towards the lymphatic nodes ${ }^{[13]}$. In the lymphatic nodes, DCs initiate immune responses by presenting antigens to lymphocytes. Importantly, DCs are a commonly recognized target for negative immunoregulation by MSCs derived from different tissues ${ }^{[14,15]}$. To the contrary, the effects of NSPCs on DCs' differentiation and maturation have not been fully disclosed.

Usually, NSPC cultures are established and maintained using serumfree media supplemented with the epidermal growth factor to keep the cells undifferentiated ${ }^{[16]}$. Nevertheless, every NSPC culture is a mix of cells at varying differentiation stages. Variability of NSPC culture composition may depend upon the source of cell material and the conditions during culture initiation and maintenance. The presented study shows that substitution of growth factors in the NSCP culture medium for fetal calf serum (FCS) can alter the in vitro effects of NSPCs upon DCs differentiation suggesting change of some of their in vivo immunomodulation properties. 


\section{Materials and methods}

\section{Cell cultures}

NSPC cultures were initiated as described elsewhere ${ }^{[17]}$ from cells of the neocortical portions of the brain of non-viable fetuses at 9-10 weeks of gestation obtained after therapeutic abortions in full accordance with the national regulations after getting patient's written consent. The work protocols were approved by the Research Center of Obstetrics, Gynecology and Perinatology (Moscow, Russia) ethical committee.

Serum-containing culture medium comprised DMEM/F12 (Gibco), 15 мM Hepes, 2 мM L-glutamine, 10\% FCS (HyClone), and antibioticantimicotic solution (Gibco). In the serum-free medium FCS was substituted for the epidermal growth factor (EGF) $-20 \mathrm{ng} / \mathrm{ml}$, basic fibroblast growth factor (FGF-2) - $20 \mathrm{ng} / \mathrm{ml}$ (both from ProSpec), heparin $-8 \mathrm{ug} / \mathrm{ml}$, and N-2 supplement (Gibco). After more than $1 / 3$ of neurospheres exceeded 250 um diameter, cell aggregates were destroyed by repetitive pipetting. Bone marrow MSC cultures were set up as described earlier ${ }^{[18]}$.

Twenty four hours before setting up the NSPCs co-culture with monocytes, neurospheres were disaggregated and issuing cells transferred to a 24 -well plate (Corning) and maintained at $37^{\circ} \mathrm{C}$ and $5 \% \mathrm{CO}_{2}$ in $0.5 \mathrm{ml}$ DMEM/F12 medium with $10 \% \mathrm{FCS}$. The following day, plated-NSPCs were treated with $10 \mu \mathrm{g} / \mathrm{ml}$ Mitomycin C (SigmaAldrich) for 2 hours and washed thrice with Hanks' solution before the addition of monocytes. Monocytes were obtained by the immunomagnetic separation of mononuclear cells utilizing Dynabeads ${ }^{\circledR}$ Untouched $^{\mathrm{TM}}$ Human Monocytes Kit (Invitrogen). The mononuclear cells were isolated from the peripheral blood by Ficoll gradient centrifugation for $30 \mathrm{~min}$ at $400 \mathrm{~g}$. Blood samples were collected from healthy donors in the Research Center of Obstetrics, Gynecology and Perinatology (Moscow, Russia) in accordance with the national regulations. Isolated monocytes were suspended in RPMI-1640 medium (Gibco) supplemented with 5\% heat inactivated FCS, $50 \mu \mathrm{g} / \mathrm{ml}$ gentamycin and $2 \mathrm{mM}$ HEPES (Stem Cells) and transferred to the wells with probed cells in the amount of $2 \times 10^{5}$ cells per well. Monocyte differentiation was induced with $80 \mathrm{ng} / \mathrm{ml}$ granulocyte macrophage colony-stimulating factor (GM-CSF) and 50 ng/ml interleukine-4 (IL-4) (both from ProSpec). After 4 days the cells were suspended and monocyte differentiation evaluated by flow cytometry.

To study NSPCs' effects on the DCs maturation DCs were first obtained by monocyte differentiation in $25 \mathrm{~cm}^{2}$ culture flasks (Corning) in the presence of GM-CSF and IL-4. In 4 days, DCs were transferred to 24 -well plates in the amount of $2 \times 10^{5}$ cells per well. The wells were pre-seeded with NSPCs as described above. Besides GM-CSF and IL-4, the co-culture medium was supplemented with 1 $\mu \mathrm{g} / \mathrm{ml}$ bacterial lipopolysaccharide (LPS; sigma-Aldrich) or $20 \mathrm{ng} / \mathrm{ml}$ tumor necrosis factor alfa (TNF-a; Prospec) and $1 \mu \mathrm{g} / \mathrm{ml}$ prostaglandin E2 (PGE2; sigma-aldrich). After 3 days DCs maturity was evaluated by flow cytometry.

\section{Immunocytochemistry}

For immunocytochemical analysis neurospheres were fixed on glass slides with paraformaldehyde; monoclonal antibodies (MoAbs, Abcam) against markers of NSPCs differentiation were added, followed by anti-species secondary antibodies (Chemicon) labeled with Rodamine or FITC. Visualization was carried out with Zeiss Axiovert 200M microscope utilizing confocal and epifluorescence regimens.

\section{Flow cytometry}

For flow cytometry analysis cells were immunostained with fluorochrome-conjugated MoAbs (BD Bioscience) against specific cellular markers. In the case of intracellular staining Cytofix/Cytoperm ${ }^{\mathrm{TM}}$ solution (BD Bioscience) was used. Fluorescence intensity and light scattering were measured with FACS-Aria flow cytometer/cell sorter (BD Bioscience).

\section{Results}

We studied 3 cultures set up and grown in serum-free medium (designated as $\mathrm{Cn}$, where " $n$ " is the autopsy number) and 4 cultures maintained in FCS-containing medium (denoted Sn). C235 and S235 cultures were raised from the same autopsy sample (Table 1).

Neurospheres maintained in FSC-containing medium looked similar to "classic" neurospheres grown in serum-free conditions (Figure 1A), although they were harder to disaggregate. If the amount of culture medium allowed free flotation of neurospheres, only a small fraction of them adhered to plastic and initiated adhesive cultures of NSPCs. Adhesive NSPCs formed clusters consisting either of cells with fibroblast-like morphology (Figure 1B), or cells with thin long processes forming web-like structures (Figure 1C), probably revealing initial stages of glial and neuronal differentiation, respectively. Both "C" and " $\mathrm{S}$ " type of neurospheres expressed early neuroblast marker $\beta$ III-tubulin. They also were positive for glial fibrillary acidic protein (GFAP), an intermediate filament protein typically expressed in astroglia (Figure 1D,E). Like "classic" neurospheres, neurospheres obtained and cultured in the presence of FCS, included cells positive for nestin, a type VI intermediate filament protein of the neuroectoderm and nerve tube neuroepithelium, as well as vimentin, another intermediate filament protein expressed in neural and reactive astrocytes precursor cells (Figure 1E). Nestin and vimentin expression was also observed during NSPCs transfer into the adhesive state (Figure 1F). A quantitative analysis of disaggregated neurospheres by flow cytometry revealed a slight increase in the expression of GFAP in cells cultured in the presence of FCS (Figure 1G). Therefore, marker expression analysis demonstrated close similarity between cellular composition of neurospheres maintained in medium with FCS and "classic" neurospheres from "C" type cultures.

\section{Table 1. NSPCs cultures studied.}

\begin{tabular}{|c|c|c|c|}
\hline Culture & $\begin{array}{c}\text { Presence of } \\
\text { FBS in culture } \\
\text { medium }\end{array}$ & $\begin{array}{c}\text { Days in culture prior } \\
\text { to co-cultivation with } \\
\text { monocytes }\end{array}$ & $\begin{array}{c}\text { Passage } \\
\text { number }\end{array}$ \\
\hline C56 & no & 48 & 5 \\
\hline C69 & no & 36 & 4 \\
\hline C235 & no & $12 ; 52$ & $0 ; 6$ \\
\hline S235 & yes & $12 ; 52$ & $0 ; 6$ \\
\hline S163 & yes & 62 & 5 \\
\hline S164 & yes & 51 & 4 \\
\hline S170 & yes & 38 & 5 \\
\hline
\end{tabular}




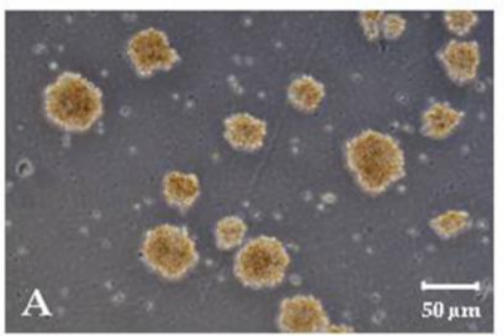

NSPC s cultured without FCS

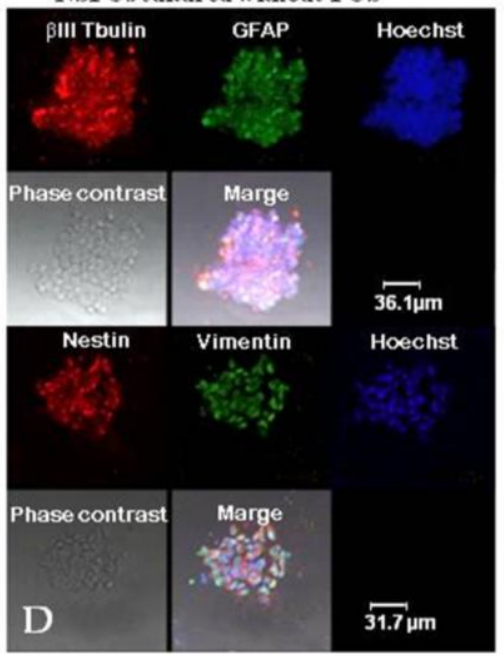

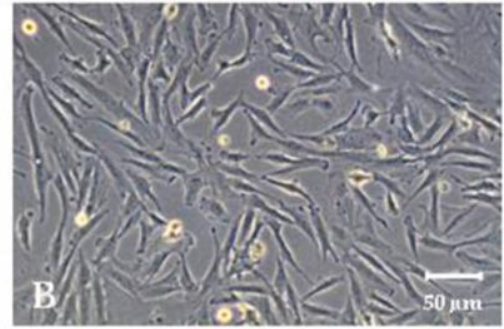

NSPC s cultured in presence of FCS

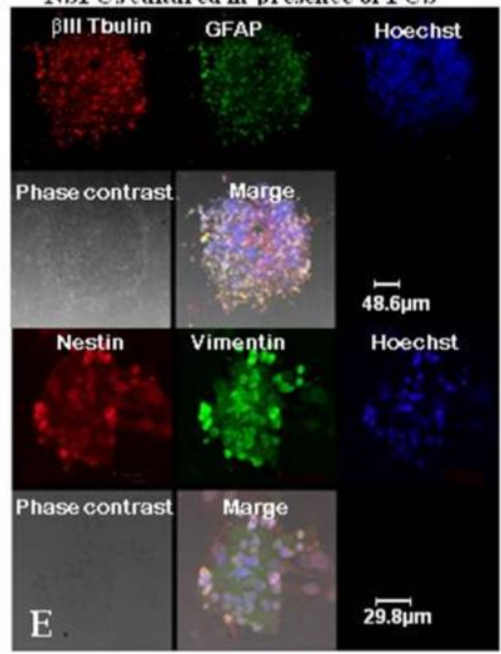

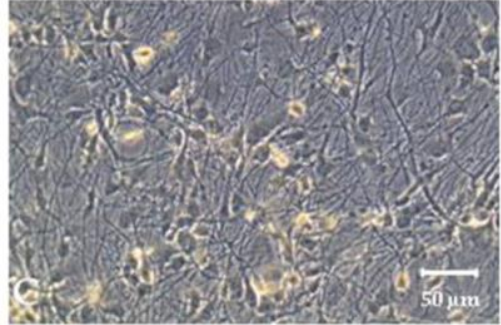
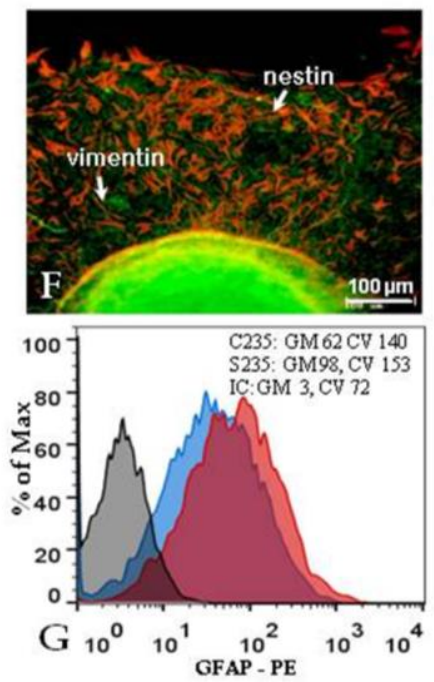

Figure 1. Neural stem/progenitor cells (NSPCs) isolated and maintained in the presence of fetal calf serum (FCS) demonstrate similarities to "classic" NSPCs grown in serumfree medium.

(A-C) Phase contrast microscopy of S170 culture in FCS-containing medium: neurospheres (A); fibroblast-like cells (B) and web-like structures (C) formed by NSPCs. (D-E) Expression of BIII Tubulin, glial fibrillary acidic protein (GFAP), nestin and vimentin by NSPCs maintained as neurospheres in serum free (D) or FCS-containing (E) medium, confocal microscopy of C235 (serum-free medium) and S235 (FCS-containing medium) cultures. (F) Expression of nestin (red) and vimentin (green) by cells relocating from neurospheres to plastic-adherent state in S170 culture grown in FCS-containing medium, fluorescent microscopy. (G) Flow cytometry analysis of GFAP expression: C235 (serumfree medium) culture- blue curve, S235 (FCS-containing medium) culture-red curve, isotype control (IC) - grey curve. Geometric means (GM) and coefficients of variation (CV) are displayed in the upper right corner.

We compared the ability of NSPCs from "S" and "C" cultures to modulate the process of monocyte differentiation to DCs. NSPCs were transferred to adhesive state and co-cultivated with peripheral blood monocytes isolated from healthy donors. Bone marrow MSCsmonocyte co-culture was used as control. Differentiation of monocytes was initiated by the addition of GM-CSF and IL-4. After 4 days in co-culture, cells were suspended, double-stained with fluorochrome-conjugated MoAbs against monocyte marker CD14 and DCs marker CD1a, and cell suspension was analyzed by flow cytometry. Differentiation efficacy was estimated by assessing the ratios of the numbers of cells expressing CD14 or CD1a. NSPCs from " $S$ " cultures established and maintained in medium with FCS effectively blocked DC differentiation from monocytes (Figure 2 A,B), while NPSCs from "C" cultures grown under "classic" serum-free conditions had no influence on DC differentiation (Figure 2C,D). This cannot be explained by the variability of the properties of cells derived from different donors, because S235 and C235 cultures prepared from the same tissue sample produced different effects (Figure 2B, D). Importantly, S235 and C235 cultures were also tested immediately after neurosphere formation, before the first passaging and at that early stage they did not affect the differentiation of DCs (result not shown). The summary of the results for all tested cultures is presented in Figure 2E. The inhibitory action of NSPCs from "S" cultures was dose-dependent and similar to the effect of bone marrow MSCs.

Flow cytometry analysis of CD83 and HLA-DR expression in all cultures presented in Table 1, demonstrated that co-culturing of immature DCs with NSPCs did not significantly alter their LPS-
(Figure 3) or TNF $\alpha$-PGE2-induced maturation irrespective of what, "S" or "C", type of NSPCs culture was examined. However, in some experiments, we detected moderate decrease in CD 83 expression within the CD 83 positive subpopulation (Figure 3C) or reduction of the quantity of cells with high for HLA-DR expression (Figure 3D).

In order to compare " $\mathrm{C}$ " and "S" cultures with respect to the types of cells they are composed of, C235 and S235 cultures derived from the same autopsy sample were stained with fluorochrome-conjugated MoAbs against a number of surface markers and analyzed by flow cytometry (Figure 4). Neither C235, nor S235 cells expressed differentiating neuroblasts and epithelial cells marker CD24 or markers of hematopoietic cells including CD34, the most common hematopoetic stem cell marker. Both types of cultures displayed relatively low staining with the antibody against CD133 stem cell marker. Since MSCs actively suppress DCs differentiation from monocytes, we estimated the likelihood of NSPCs conversion to MSCs-like phenotype in serum-containing medium and found it implausible, since we failed to find any cells positive for MSCsassociated CD104 (endoglin) or CD54 (ICAM-1) among S235 cells. CD90 (Thy-1), a glycoprotein present in the plasma membranes of both MSCs and neurons was weakly expressed only by C235 NSPCs suggesting their readiness for neurogenic differentiation. Unlike C235, S235 culture contained a small subset of cells expressing CD44 and an even smaller subpopulation of cells positive for CD73. Though the assortment of cell types present in NSPC cultures grown in the presence or absence of FCS may be slightly different, the difference seems too small to define NSPCs' suppressive effects on the immune system. 


\section{NSPCs cultured in presence of FCS}

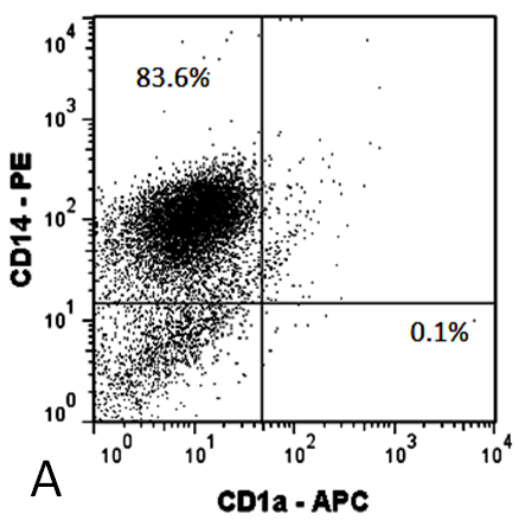

NSPCs cultured without FCS

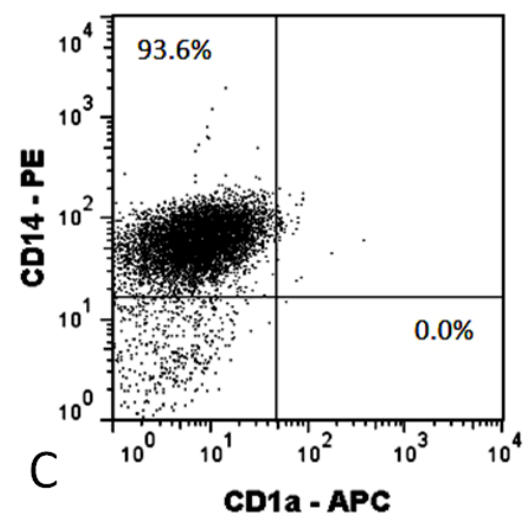

NSPCs cultured in presence of FCS

+ GM-CSF, IL-4

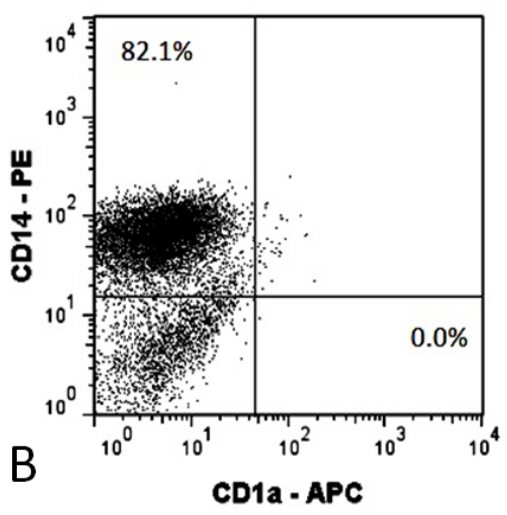

NSPCs cultured without FCS + GM-CSF, IL-4

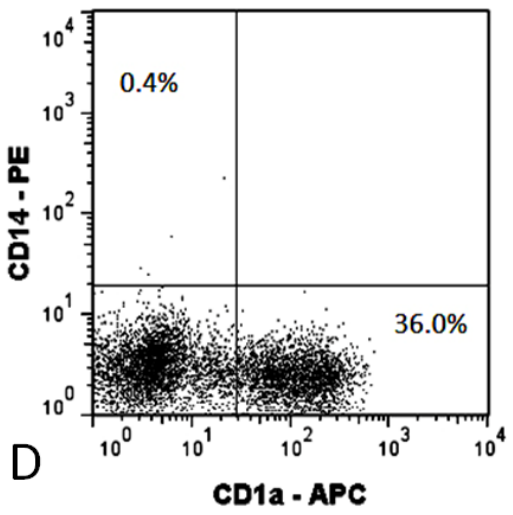

E

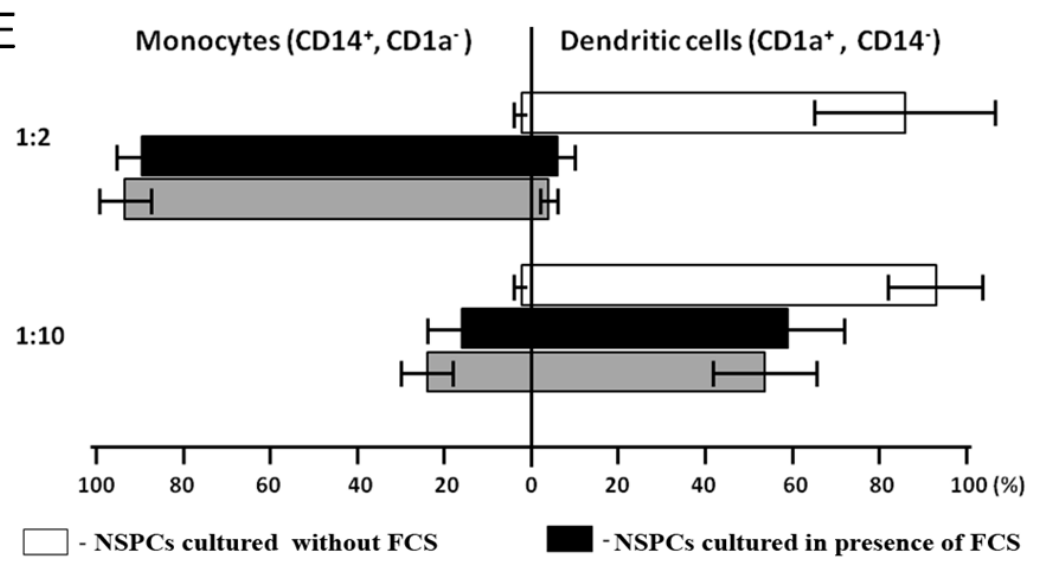

MSC

Figure 2. Effects of neural stem/progenitor cells (NSPCs) grown with or without fetal calf serum (FCS) upon differentiation of dendritic cells (DCs) from monocytes.

Co-cultivation of NSPCs from FCS-containing S235 culture (A, B) or FCS-free C235 culture (C, D) with monocytes at 1:2 NSPC/monocyte ratio in the absence (A, C) or presence (B, D) of differentiation factors: granulocyte macrophage colony-stimulating factor (GM-CSF) and interleukine-4 (IL-4). After 4 days in co-culture the cells were suspended, double stained with fluorescent monoclonal antibodies (MoAbs) against monocyte marker CD14 and DC marker CD1a and analyzed by flow cytometry. (E) Combined data for 3 "C" type (serum-free medium) cultures and 4 "S" type (FCS-containing medium) NSPCs cultures. Co-cultures with mesenchymal stem cells (MSCs) were used as positive control. Left-side columns: relative percentage of remaining monocytes calculated by formula: $\mathrm{C} / \mathrm{M} \times 100 \%$ (C - percentage of CD14 positive monocytes in co-culture, $\mathrm{M}$ - percentage of CD14 positive monocytes in co-culture without GM-CSF and IL-4); right-side columns: relative percentage of emerging DCs calculated by formula: $\mathrm{C} / \mathrm{M} \times 100 \%$ (C - percentage of CD1a positive DCs in co-culture, $\mathrm{M}$ - percentage of CD1a positive DCs in monoculture). Ratios of effector cells and monocytes in the co-culture well shown on the left. Percentages of both monocytes and DCs significantly differ between serum-free NSPCs cultures and FCS-containing NSPCs cultures. Mann Whitney statistical test was used to test for statistical significance, $\mathrm{P}<0.05$ ). 


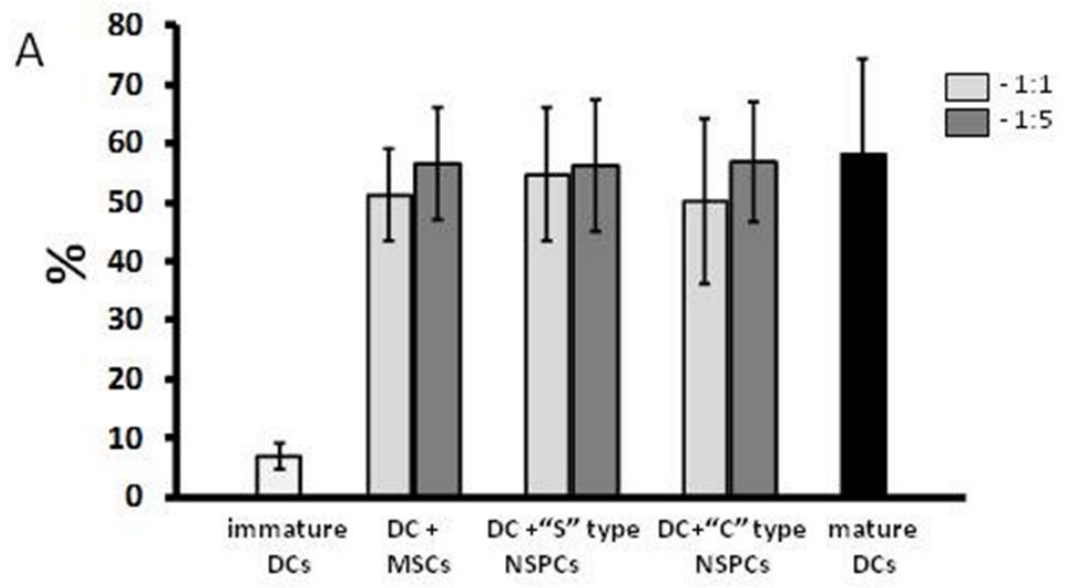

B
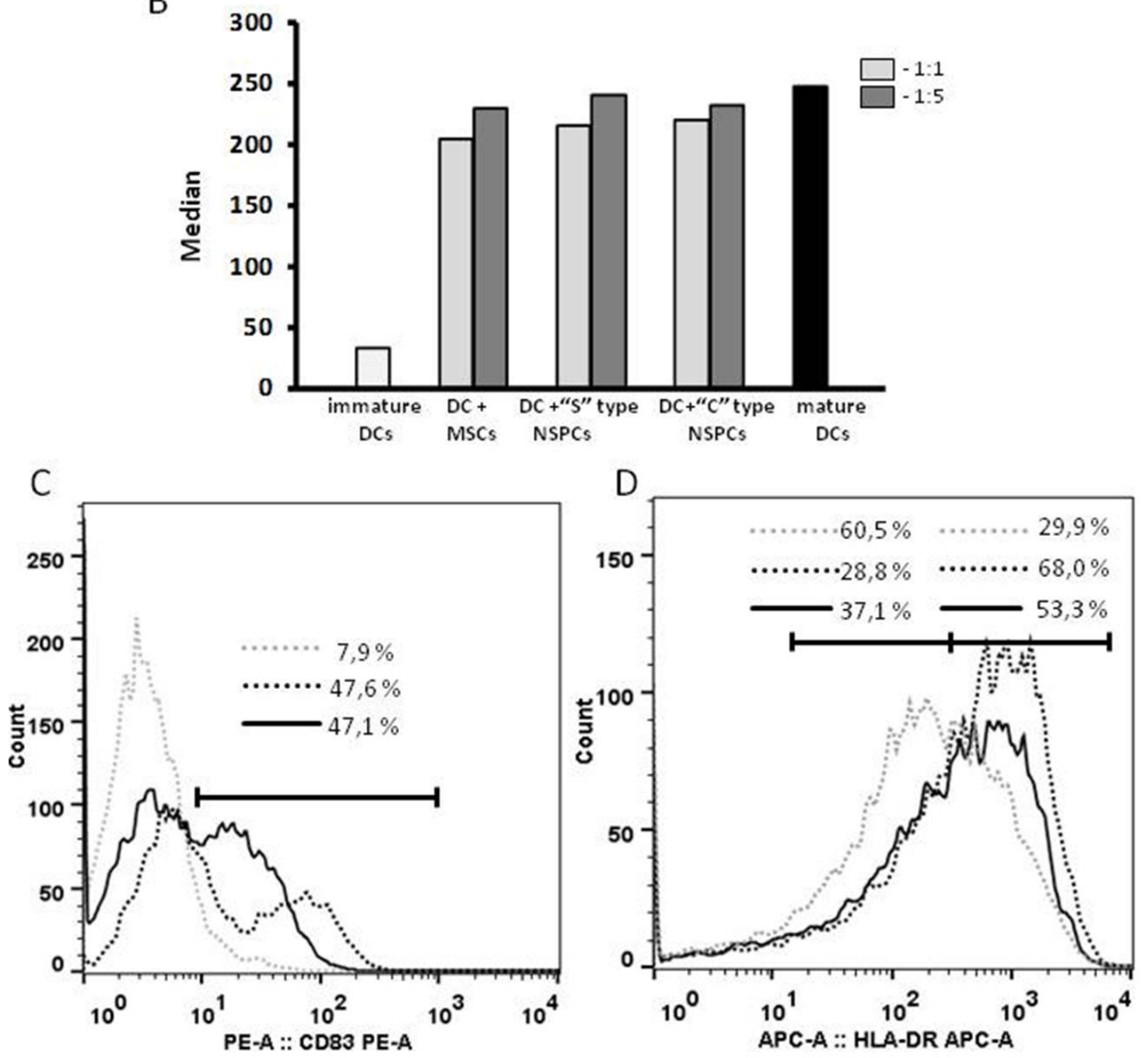

Figure 3. Effects of neural stem/progenitor cells (NSPCs) grown with or without fetal calf serum (FCS) upon maturation of dendritic cells (DCs). NSPCs were co-cultivated with immature DCs at 1:1 and 1:5 NSPCs/DCs ratios in the presence of bacterial lipopolysaccharide (LPS). After 3 days in co-culture the cells were suspended, double stained with fluorescent monoclonal antibodies (MoAbs) against CD83 and HLA-DR and analyzed by flow cytometry. (A, B) Combined data for 3 " $C$ " type (serum-free medium) and 4 "S" type (FCS-containing medium) NSPCs cultures. (A) Percentage of DCs expressing CD83. (B) Median of fluorescence intensity of MoAbs against HLA-DR. (C, D) Histograms showing CD $83(C)$ and HLA-DR (D) expression level for DCs co-cultured with S163 (representative experiment). Black curves - C163 co-culture; black broken curves - MSCs co-culture; grey broken curves - DCs monoculture without LPS. The percentages of cells within the defined populations given on top. 

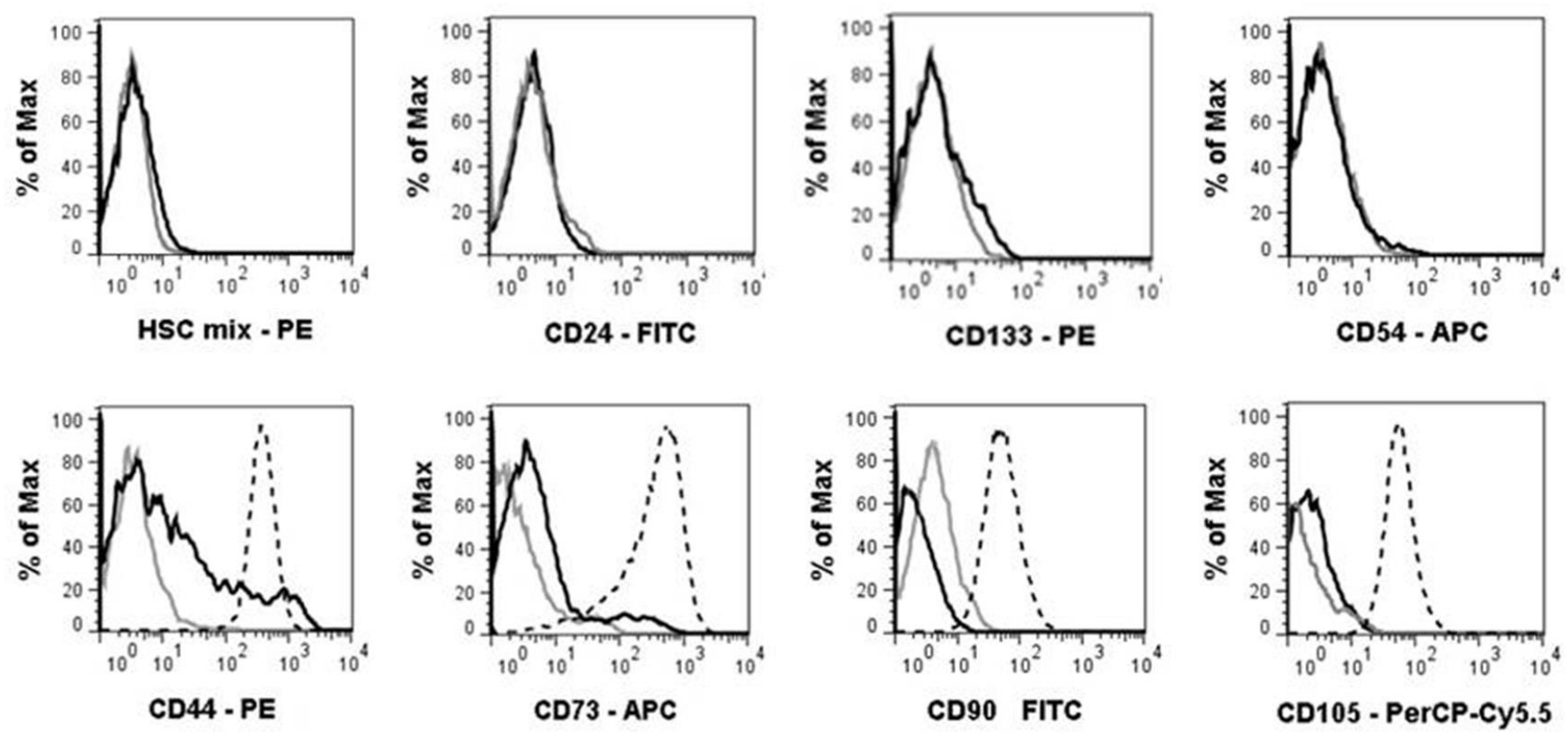

\begin{abstract}
Figure 4. Expression of surface markers by neural stem/progenitor cells (NSPCs) from serum-containing and serum-free cultures derived from the same autopsy specimen.

Cell suspensions from S235 and C235 cultures were treated with fluorochrome-conjugated monoclonal antibodies (MoAbs) and analyzed by flow cytometry. Black curves - S235; grey curves - C235; broken curves - mesenchymal stem cells (MSCs). HSC mix - fluorochrome-conjugated MoAbs cocktail against hematopoietic markers: CD19, CD11b, CD34, CD45, HLA-DR.
\end{abstract}

\title{
Discussion
}

Our data suggest that the presence or absence of FCS in the culture medium is crucially important for the formation of two alternate NSPC phenotypes ("S" and "C" phenotypes, respectively) characterized by the ability to block DCs differentiation from monocytes. The short time exposure to FCS is probably not sufficient for the formation of "S" type cultures, because " $S$ " phenotype is established only after passaging of NSPCs culture, as seen from our experiments with C235 and S235 cultures. It is not yet clear if DCs' differentiation block is induced by the majority of cells in NSPCs " $\mathrm{S}$ " cultures or by a minor subpopulation.

It was suggested that serum exposure promotes NSPCs differentiation into astroglia ${ }^{[16]}$. Subsequently, gene expression profiling of human NSPCs following their serum-induced astrocyte differentiation revealed elevated levels of the expression of 45 genes including $\mathrm{GFAP}^{[19]}$. Moreover, astrocytes can suppress in vitro monocyte/microglial activation and function ${ }^{[20]}$. Therefore, it seems likely that NSPCs' ability to control DCs differentiation may be associated with their serum-induced differentiation into astrocytes direction. However, this suggestion is opposed by certain results of our current studies. Here, as well as in our earlier publication we showed that NSPCs maintained in FSC-containing medium supposed to promote astrocytic diffentiation, still capable of expressing such "stemness" markers as nestin and Lex/SSEA1, as well as an early marker of neuronal differentiation BIII-tubulin. Their expression is mostly localized in neurosphere-like floating cell aggregates ${ }^{[21]}$. Probably, maintaining of NSPCs cultures in serum-containing media results in the accumulation of the least differentiated cells in neurospheres, while more differentiated cells adhere to cultural plastic and are eliminated at passaging.
Data concerning the interactions of NSPCs with DCs obtained by other researchers are scarce. On one hand, NSPCs derived by differentiation of human embryonic stem cells had almost no effect on monocytes differentiation in vitro ${ }^{[22]}$. On the other, human fetal NSPCs grown in serum-free conditions inhibited differentiation of monocytes into DCs ${ }^{[23]}$. This discrepancy may be caused by various reasons including the differences of cellular composition of the tested cultures and culture conditions. Data presented here show that NSPCs' impact on DCs differentiation from monocytes depends on the culture conditions of NSPCs.

The mechanism of FCS effect and difference between " $\mathrm{C}$ " and " $\mathrm{S}$ " phenotypes need further investigation including the possible role of BMP4 and other candidate factors on NSPCs and their co-cultures with monocytes. It is obvious, though, that culture conditions can result in differences in cell phenotypes. Importantly, dependence of essential cell properties from culture conditions can greatly complicate the process of development of cell-based therapies. Manufacture of clinical-grade cell products involves adaptation of laboratory culture methods, including drastic increase of cell production and exclusion of xenogeneic proteins achieved by substitution of FCS for human blood serum or utilizing serum-free media. That means that the results of basic and preclinical research carried out with cells grown using common laboratory methods, in some cases can be irrelevant to the effects of the controlled quality cell product obtained in a GMP facility and used for clinical studies. Our results show yet again that all preclinical studies with cultured cells have to be conducted using cells produced in exactly the same conditions and with the same equipment as the future clinical grade product. Dependence of clinically relevant properties of cell therapy 
products from the presence of certain factors, including serum components in the culture medium suggests that the effects of cell transplantation may depend upon the administration route. Really, after intravenous or intra-arterial administration transplanted cells find themselves in direct contact with all serum components, while after intracutaneous injection cells find themselves in a dramatically different environment.

\section{Conclusion.}

Addition of FCS instead of conventional combinations of growth factors to the NSPCs culture medium results in substantial modification of the immunological properties of the cultured cells manifested as the ability to suppress the in vitro differentiation of DCs from peripheral blood monocytes. At the same time, the patterns of cell types constituting the culture are similar in the presence or absence of FCS. In a broader prospective, it means that cells with almost identical phenotypes can display opposite immunomodulation properties. It should be taken into account when developing NSPCsbased cell products for regenerative medicine.

\section{References}

1. Andres RH, Horie N, Slikker W, Keren-Gill H, Zhan K, Sun G, Manley NC, Pereira MP, Sheikh LA, McMillan EL, Schaar BT, Svendsen CN, Bliss TM, Steinberg GK. Human neural stem cells enhance structural plasticity and axonal transport in the ischaemic brain. Brain. 2011; 134(Pt 6): 1777-89.

2. Lee ST, Chu K, Jung KH, Kim SJ, Kim DH, Kang KM, Hong NH, Kim JH, Ban JJ, Park HK, Kim SU, Park CG, Lee SK, Kim M, Roh JK. Anti-inflammatory mechanism of intravascular neural stem cell transplantation in haemorrhagic stroke. Brain. 2008; 131(Pt 3): 616-29.

3. Pluchino S, Furlan R, Martino G. Cell-based remyelinating therapies in multiple sclerosis: evidence from experimental studies. Curr. Opin.Neurol. 2004; 17(3): 247-55.

4. Ottoboni L, De Feo D, Merlini A, Martino G. Commonalities in immune modulation between mesemchymal stem cells (MSCs) and neural stem/precursor cells (NPCs). Immunol Lett. 2015; 168(2): 228-39.

5. Pluchino S, Zanotti L, Rossi B, Brambilla E, Ottoboni L, Salani G, Martinello M, Cattalini A, Bergami A, Furlan R, Comi G, Constantin G, Martino G. Neurosphere-derived multipotent precursors promote neuroprotection by an immunomodulatory mechanism. Nature. 2005; 436(7048): 266-71.

6. Ben-Hur T. Immunomodulation by neural stem cells. J Neurol Sci. 2008; 265(1-2): 1024.

7. Pluchino S, Zanotti L, Brambilla E, Rovere-Querini P, Capobianco A, Alfaro-Cervello C, Salani G, Cossetti C, Borsellino G, Battistini L, Ponzoni M, Doglioni C, Garcia-Verdugo JM, Comi G, Manfredi AA, Martino G. Immune Regulatory Neural Stem/Precursor Cells Protect from Central Nervous System Autoimmunity by Restraining Dendritic Cell Function. PLoS One. 2009; 4(6): e5959.

8. Fainstein N, Vaknin I, Einstein O, Zisman P, Ben Sasson SZ, Baniyash M, Ben-Hur T. Neural precursor cells inhibit multiple inflammatory signals. Mol Cell Neurosci. 2008; 39(3): 335-41.
9. Einstein O, Fainstein N, Vaknin I, Mizrachi-Kol R, Reihartz E, Grigoriadis N, Lavon I, Baniyash M, Lassmann H, Ben-Hur T. Neural precursors attenuate autoimmune encephalomyelitis by peripheral immunosuppression. Ann Neurol. 2007; 61(3): 209-18.

10. Steptoe RJ, Thomson AW. Dendritic cells and tolerance induction. Clin Exp Immunol. 1996; 105(3): 397-402.

11. Dauer M, Obermaier B, Herten J, Haerle C, Pohl K, Rothenfusser S, Schnurr M, Endres S, Eigler A. Mature dendritic cells derived from human monocytes within 48 hours: a novel strategy for dendritic cell differentiation from blood precursors. J. Immunol. 2003; 170(8): 4069-76.

12. Karalkin PA, Lupatov AY, Yarygin KN. Endocytosis of microand nanosized particles by human dendritic cells. Biochemistry (Moscow) Supplement Series A: Membrane and Cell Biology. 2009; 3(4): 410-16.

13. Christopherson K, Hromas R. Chemokine regulation of normal and pathologic immune responses. Stem Cells. 2001; 19(5): 38896.

14. Jiang XX, Zhang Y, Liu B, Zhang SX, Wu Y, Yu XD, Mao N. Human mesenchymal stem cells inhibit differentiation and function of monocyte-derived dendritic cells. Blood. 2005; 105(10): 4120-26.

15. Nauta AJ, Kruisselbrink AB, Lurvink E, Willemze R, Fibbe WE. Mesenchymal stem cells inhibit generation and function of both CD34-derived and monocyte-derived dendritic cells. J. Immunol. 2006; 177(4): 2080-87.

16. Reynolds BA. Weiss S. Clonal and population analyses demonstrate that an EGF-responsive mammalian embryonic CNS precursor is a stem cell. Dev Biol. 1996; 175 (1): 1-13.

17. Poltavtseva RA, Revishchin AV, Aleksandrova MA, Korochkin LI, Viktorov IV, Sukhikh GT. Neural stem and progenitor cells of human embryos and fetuses as a basis of new biomedical technologies. Ontogenez 2003; 34 (3): 211-15

18. Lupatov AY, Karalkin PA, Suzdaltseva YG, Burunova VV, Yarygin VN, Yarygin $\mathrm{KN}$. Cytofluorometric analysis of phenotypes of human bone marrow and umbilical fibroblast-like cells. Bull Exp Biol Med. 2006; 142 (4): 521-26.

19. Obayashi S, Tabunoki H, Kim SU, Satoh J. Gene expression profiling of human neural progenitor cells following the seruminduced astrocyte differentiation. Cell Mol Neurobiol. 2009; 29 (3): 423-38.

20. Kostianovsky AM, Maier LM, Anderson RC, Bruce JN, Anderson DE. Astrocytic regulation of human monocytic/microglial activation. J Immunol. 2008; 181(8): 5425-32.

21. Aleksandrova MA, Poltavtseva RA, Marei MV, Sukhikh GT. Analysis of Neural Stem Cells from Human Cortical Brain Structures In Vitro. Bull Exp Biol Med. 2016; 161(1): 197-208.

22. Shahbazi M, Kwang TW, Purwanti YI, Fan W, Wang S. Inhibitory effects of neural stem cells derived from human embryonic stem cells on differentiation and function of monocyte-derived dendritic cells. J Neurol Sci. 2013; 330: 85-93.

23. Pluchino S, Gritti A, Blezer E, Amadio S, Brambilla E, Borsellino G, Cossetti C, Del Carro U, Comi G, Hart B, Vescovi A, Martino G. Human neural stem cells ameliorate autoimmune encephalomyelitis in non-human primates. Ann Neurol. 2009; 66 (3): 343-54. 
Lupatov AYu, et al. J Stem Cells Regen Med 2017; 13(2)

\section{Abbreviations}

NSPCs: Neural stem/progenitor cells

DCs: Dendritic cells

MSCs: Mesenchymal stem cells

MHC: Major histocompatibility complex

FCS: $\quad$ Fetal calf serum

EGF: $\quad$ Epidermal growth factor

FGF-2: $\quad$ Basic fibroblast growth factor

GM-CSF: Granulocyte macrophage colony-stimulating factor

IL-4: Interleukine-4

LPS: Lipopolysaccharide

TNF- $\alpha$ : Tumor necrosis factor- $\alpha$

MoAbs: Monoclonal antibodies

GFAP: Glial fibrillary acidic protein

\section{Potential Conflicts of Interests}

None

\section{Acknowledgements}

This research work was supported by grant No 14-25-00179 from Russian Science Foundation.

The date analysis and preparation of the manuscript was performed within the framework of the Program for Basic Research of State Academies of Sciences for 2013-2020. The authors are grateful to Alexander Samokhin for technical assistance.

\section{Ethical standards}

The work protocols were approved by the Research Center of Obstetrics, Gynecology and Perinatology ethical committee in full accordance with the national regulations.

\section{Corresponding Author}

Alexey Yu Lupatov, Institute of Biomedical Chemistry, Pogodinskaya str. 10, 119121 Moscow, Russia, alupatov@inbox.ru. 